\title{
Obesidade em adultos de segmentos pauperizados da sociedade
}

\section{Obesity in adults from impoverished segments of the society}

Sheila Pita MARINHO ${ }^{1}$

Ignez Salas MARTINS²

José Paulo Pires PERESTRELO ${ }^{1}$

Denize Cristina de OLIVEIRA ${ }^{3}$

\section{R E S U M O}

0 aumento da incidência de obesidade em segmentos pauperizados da sociedade levanta a questão sobre sua etiologia. Este trabalho pretende caracterizar a prevalência de sobrepeso e obesidade nestas populações. Participaram da pesquisa 390 famílias, incluindo 782 indivíduos com idades iguais ou maiores de 19 anos, dos quais $360(46,0 \%)$ eram homens e $422(54,0 \%)$ mulheres. As prevalências de obesidade entre os homens das zonas urbana e rural foram de 5,6\% e 5,8\% , respectivamente. Em relação ao sobrepeso, esta incidência foi de cerca de $25 \%$ em ambas as regiões, evidenciando-se aumento com a idade. As prevalências de obesidade entre homens e mulheres foram, respectivamente, de 5,6\% e 21,6\% ( $p<0,05)$. Entre as mulheres a ocorrência de obesidade aumentou discretamente com a idade, com prevalências padronizadas de $19,8 \%$ na zona urbana e $23,4 \%$ na rural. Considerando tratar-se de uma população pauperizada, o excesso de peso, que atinge principalmente as mulheres, tem etiologia complexa e precisa ser melhor estudado.

Termos de indexação: obesidade, sobrepeso, exclusão social, adulto, população periférica.

\section{A B S T R A C T}

In impoverished segments of the society, the increase in the incidence of obesity raises a question concerned with its etiology. The objective of this paper is to characterize the prevalences of overweight and obesity in these populations. Three hundred and ninety families joined the program, including 782 individuals with

\footnotetext{
1 Mestrandas, Departamento de Nutrição, Faculdade de Saúde Pública, Universidade de São Paulo, Av. Dr. Arnaldo, 715, 01246-904, São Paulo, SP, Brasil. Correspondência para/Correspondence to: S.P.MARINHO. E-mail: sheilapita@bol.com.br

2 Departamento de Nutrição, Faculdade de Saúde Pública, U niversidade de São Paulo.

3 Departamento de Saúde Ambiental, Faculdade de Saúde Pública, Universidade de São Paulo.
} 
ages above 19 years, $360(46,0 \%)$ of them were men and $422(54,0 \%)$ were women. The prevalences of obesity among men from urban and rural areas were $5.6 \%$ and $5.8 \%$, respectively. Concerning overweight, this incidence was about $25 \%$ for both regions, and the percentages increased with age. The prevalences of obesity among men and women were, respectively, $5.6 \%$ and $21.6 \%(p<0,05)$. Among women the ocurrence of obesity increased slightly with age, and the prevalences were $19.8 \%$ in urban areas and $23.4 \%$ in rural areas. Therefore, as the evaluated population lives in extreme poverty, the excess of weight, which hits mainly the women, has a complex etiology and needs to be better studied.

Index terms: obesity, overweight, social exclusion, adult, peripheric population.

\section{N T R O D U Ç Ã O}

Estudos epidemiológicos sobre estado nutricional demonstram que o excesso de peso se apresenta como grave problema de Saúde Pública nas sociedades afluentes e nos países em desenvolvimento.

Trabalhos procurando relacionar o excesso de peso (IMC $\geq 25,0 \mathrm{~kg} / \mathrm{m}^{2}$ e $\left.<30 \mathrm{~kg} / \mathrm{m}^{2}\right)$ e/ou a obesidade (IMC $\left.\geq 30 \mathrm{~kg} / \mathrm{m}^{2}\right)$ com níveis socioeconômicos - geralmente definidos pelos indicadores escolaridade, renda e ocupação - têm revelado nas sociedades afluentes uma correlação negativa dessas morbidades com o status social, principalmente entre as mulheres ${ }^{1-4}$.

De acordo com duas grandes pesquisas realizadas no Brasil, o Estudo Nacional da Despesa Familiar, realizado em $1975^{5}$, e a Pesquisa Nacional sobre Saúde e Nutrição (PNSN), em $1989^{6}$, no intervalo de tempo entre o primeiro e o segundo levantamento, o sobrepeso aumentou na proporção de $58 \%$ para os homens e $42 \%$ para as mulheres, e 0 aumento da obesidade foi de $100 \%$ para o sexo masculino e $70 \%$ para 0 feminino. As Regiões Sul e Sudeste apresentaram as maiores prevalências, enquanto no Nordeste encontrou-se menor porcentagem. Traduzindo-se essas taxas em números, registra-se, no ano de 1989, 27 milhões de pessoas com excesso de peso. Na zona urbana o excesso de peso guarda relação direta com o nível socioeconômico entre os homens e inversa entre as mulheres. Essa tendência foi encontrada em grupamentos definidos, segundo nível socioeconômico, em estudo realizado na Área Metropolitana de São
Paulo7. Para a zona rural, entretanto, a PNSN demonstrou que a obesidade guarda relação direta com a renda familiar, em ambos os sexos.

A Pesquisa Nacional sobre Padrões de Vida, abrangendo os habitantes do Nordeste e do Sudeste do país, mostrou, tomando-se como base dados da PNSN de19896 para essas duas Regiões, aumento da prevalência da obesidade. No caso da população adulta masculina, essa elevação foi maior na Região Nordeste $(95 \%)$ do que na Sudeste (38\%). Também no caso da população adulta feminina, o aumento foi mais expressivo na Região Nordeste (60\%) em relação à Sudeste $(12 \%)^{8}$.

As explicações dadas pelos epidemiologistas para o crescimento acelerado da obesidade nas populações apontam a modernização das sociedades, a qual, entre outras coisas, provocou maior oferta de alimentos, aliada à melhoria dos instrumentos de trabalho, como a mecanização e automação. A economia de gasto de energia humana no trabalho e a maior oferta de alimentos mudaram radicalmente 0 modo de viver. 0 sedentarismo, concomitantemente à mudança na alimentação denominada de "transição nutricional" caracterizada pelo aumento no consumo de gorduras, açúcar e cereais refinados e pela redução no consumo de carboidratos complexose fontes de fibras - mudou o perfil de morbimortalidade nas sociedades, destacando-se 0 excesso de peso e a obesidade como doenças fundamentais. Por essas razões, a obesidade tem sido denominada como "doença da civilização" ou "síndrome do novo mundo" 9-11. 
Por outro lado, tem sido sugerido em extensa literatura que a etiologia da obesidade é bastante complexa. A teoria da transição nutricional procura explicá-la como resultante de mudanças nos padrões alimentares aliadas ao desenvolvimento tecnológico, o qual teria levado o homem ao menor dispêndio energético na sua sobrevivência. Assim, dietas com alto conteúdo energético e o sedentarismo estariam na etiologia da obesidade. Esta teoria se aplica satisfatoriamente na explicação da evolução da obesidade e dos hábitos alimentares no Brasil nas últimas décadas ${ }^{10}$

Conforme sugerem alguns desses estudos, famílias mais pobres tendem a consumir dietas de alta densidade energética, por serem mais baratas. Ao mesmo tempo, seu lazer, atualmente, restringe-se praticamente a assistir programas de televisão ${ }^{11}$, tornando-as sedentárias. Entretanto, ao se deparar com populações abaixo da "linha da pobreza", outros elementos teriam de ser considerados, como, por exemplo, a possibilidade de adaptações metabólicas diante da escassez crônica de consumo energético, sugerida por Velasquéz et al. (1999)12 em pesquisa realizada em obesas de baixa estatura.

Mondini (1996) ${ }^{13}$ enfatiza o quanto é equivocada a afirmação de que a obesidade prevaleceria entre os grupos de maior poder aquisitivo. Em estudo sobre a distribuição da desnutrição e da obesidade, com base em dados obtidos pela PNSN, observou prevalência elevada de excesso de peso nas classes de baixa renda, principalmente entre as mulheres; nos grupamentos com até 0,25 salários mínimos per capita, aproximadamente $13 \%$ delas apresentavam Índice de Massa Corporal acima de $27,7 \mathrm{~kg} / \mathrm{m}^{2}$, correspondendo ao percentil 95.

Com base nos achados de Mondini $(1996)^{13}$, pode-se questionar a real dimensão do problema da obesidade em populações pobres. Faz-se necessário, portanto, realizar outros estudos que dêem subsídios para pesquisas sobre a etiologia da obesidade em segmentos de baixo nível socioeconômico.
Considerando o exposto, o objetivo deste trabalho é caracterizar a prevalência da obesidade em adultos pertencentes a famílias com renda mensal de até dois salários mínimos, discutindo, quando possível, alguns de seus determinantes.

\section{CASUÍSTICA E MÉTODOS}

Esta pesquisa faz parte de um projeto desenvolvido em julho de 1997 com a finalidade de investigar a situação de saúde, educação e trabalho em populações pauperizadas, visando fornecer subsídios a programas de intervenção voltados à geração de empregos em municípios integrantes do Programa Comunidade Solidária do Governo Federal. 0 critério do Programa na escolha dos municípios a serem contemplados com a distribuição de alimentos era o de ter até 40 mil habitantes, cerca de $25 \%$ da população com renda familiar mensal menor ou igual a dois salários mínimos (cerca de 0,4 salários mínimos per capita) e baixo índice de desenvolvimento humano. No Estado de São Paulo 144 municípios estão nessas condições ${ }^{14}$. Dentre eles foram escolhidos dois, M onteiro Lobato e Santo Antônio do Pinhal, para um estudo piloto abrangendo as famílias cadastradas no Programa. Ressalta-se que a escolha destas cidades e das famílias contempladas com a cesta de alimentos deu-se, unicamente, porque o objetivo deste trabalho era atingir a população pauperizada ${ }^{15}$.

Ao todo foram identificadas 495 famílias com 995 indivíduos acima de 19 anos, sendo 488 (49\%) homens e 507 (51\%) mulheres. Aderiram à pesquisa 390 famílias, com $360(46 \%$ ) homens e 422 (54\% ) mulheres. Encontrou-se dificuldade na adesão dos homens à pesquisa, em parte devido à resistência em se submeter ao exame clínico e, por outro lado, em virtude da grande mobilidade espacial da população masculina mais jovem, formada fundamentalmente por trabalhadores diaristas. Foram realizadas entrevistas domiciliares, de caráter socio demográfico, sobre condições de saúde, trabalho, ocupação, moradia e saneamento ambiental. Após a entrevista os indivíduos foram convocados para o 
exame clínico, geralmente realizado em escolas ou centros de saúde.

O padrão de referência usado para a definição de estado nutricional foi o Índice de Massa Corporal (IMC) (peso/altura ${ }^{2}$ ), com os pontos de corte propostos pela World Health Organization (WHO) $)^{11}$. Ou seja, valores $<18,5 \mathrm{~kg} / \mathrm{m}^{2}$ correspondem à desnutrição; entre 18,5 e $24,9 \mathrm{~kg} / \mathrm{m}^{2}$, à normalidade; de 25,0 a $29,9 \mathrm{~kg} / \mathrm{m}^{2}$, à pré-obesidade; iguais ou superiores a $30,0 \mathrm{~kg} / \mathrm{m}^{2}$, à obesidade. As pessoas, com roupas leves e sem sapatos, foram pesadas em balança digital Filizola, com capacidade de até $150 \mathrm{~kg}$ e precisão de $100 \mathrm{~g}$. A altura foi medida com fita métrica inextensível, afixada na parede, e um esquadro. Os indivíduos foram medidos descalços, em posição ereta, de forma a manter o chamado "plano de Frankfurt", com as costas e a parte posterior dos joelhos encostadas à parede.

Os dados coletados foram tabulados no programa para análise epidemiológica Epi Info.

A comparação entre grupos foi feita através da prevalência padronizada de acordo com a idade, utilizando-se como população padrão a média harmônica das duas populações avaliadas, a urbana e a rural. A comparação entre as prevalências foi feita pelo teste de diferença de proporções.

\section{RESULTA D O S}

Avaliou-se o estado nutricional dos homens das zonas urbana e rural, segundo faixa etária (Tabela 1). Para os indivíduos da zona urbana com idade entre 20 e 39 anos a prevalência de obesidade foi de $1,4 \%$. Para aqueles entre $40 \mathrm{e}$ 59 anos essa taxa foi de $7,1 \%$, e entre os maiores de 60 anos ela foi de $14,8 \%$. Tanto a prevalência bruta quanto a padronizada para a idade foram de 5,6\%. O sobrepeso situou-se em torno de $23,0 \%$ nas faixas etárias de 20 a 39 e de 40 a 59 anos, aumentando para 33,3\% para os idosos.

$\mathrm{Na}$ zona rural, a proporção de obesos foi inferior a 10,0\% em todas as faixas etárias. Além disso, a prevalência de obesidade entre os idosos foi consideravelmente menor, com diferenças estatisticamente significantes em relação à zona urbana $(Z 0=3,20 ; p<0,05)$. As prevalências de obesidade bruta e padronizada para a idade foram de $6,0 \%$ e $5,8 \%$, respectivamente. $A$ pré-obesidade atingiu cerca de $17,0 \%$ dos indivíduos abaixo de 40 anos da zona rural, aumentando expressivamente nas idades mais avançadas.

Com relação à analise do estado nutricional das mulheres (Tabela 2), na zona urbana a prevalência de obesidade aumentou discreta-

Tabela 1. Número e porcentagem de homens segundo índice de massa corporal, zona de residência e idade. Municípios de Monteiro Lobato e Santo Antônio do Pinhal, 1997.

\begin{tabular}{|c|c|c|c|c|c|c|c|c|c|c|}
\hline \multirow{3}{*}{ Categoria } & \multicolumn{6}{|c|}{ Anos } & \multicolumn{4}{|c|}{ Total } \\
\hline & \multicolumn{2}{|c|}{$20-39$} & \multicolumn{2}{|c|}{$40-59$} & \multicolumn{2}{|c|}{$\geq 60$} & \multicolumn{2}{|r|}{$\mathrm{PB}^{1}$} & \multirow{2}{*}{$\begin{array}{c}\mathrm{PP}^{2} \\
\%\end{array}$} & \multirow{2}{*}{$\frac{\text { IC95 (PP) }}{\%}$} \\
\hline & $\mathrm{n}$ & $\%$ & $\mathrm{n}$ & $\%$ & $\mathrm{n}$ & $\%$ & $\mathrm{n}$ & $\%$ & & \\
\hline \multicolumn{11}{|l|}{ Urbana } \\
\hline Sem excesso IMC $<25$ & 56 & 75,7 & 29 & 69,0 & 14 & 51,9 & 99 & 69,2 & 69,4 & $(62,5-76,3)$ \\
\hline Pré-obesidade $25,0>=\mid M C<30,0$ & 17 & 23,0 & 10 & 23,8 & 9 & 33,3 & 36 & 25,2 & 25,1 & $(18,6-31,6)$ \\
\hline Obesidade $\mid \mathrm{M} C>=30$ & 1 & 1,4 & 3 & 7,1 & 4 & 14,8 & 8 & 5,6 & 5,6 & $(2,0-9,2)$ \\
\hline Subtotal & 74 & 51,7 & 42 & 29,4 & 27 & 18,9 & 143 & & & \\
\hline \multicolumn{11}{|l|}{ Rural } \\
\hline Sem excesso IMC $<25$ & 82 & 78,1 & 45 & 58,4 & 21 & 60,0 & 148 & 68,2 & 68,7 & $(61,8-75,6)$ \\
\hline Pré-obesidade $25,0>=\mid \mathrm{M} \mathrm{C}<30,0$ & 18 & 17,1 & 25 & 32,5 & 13 & 37,1 & 56 & 25,8 & 25,5 & $(19,0-32,0)$ \\
\hline Obesidade $\mid \mathrm{M} C>=30$ & 5 & 4,8 & 7 & 9,1 & 1 & 2,9 & 13 & 6,0 & 5,8 & $(2,2-9,4)$ \\
\hline Subtotal & 105 & 48,4 & 77 & 35,5 & 35 & 16,1 & 217 & & & \\
\hline
\end{tabular}

Nota: (1) PB = Prevalência Bruta; (2) PP = Prevalência padronizada; (3) IC95 = Intervalo de confiança de 95\%. 
mente com a idade, com percentuais em torno de $20,0 \%$; contudo, a ocorrência de sobrepeso aumentou acentuadamente de $26,0 \%$, entre 20 e 39 anos, para 44,0\%, a partir dos 40 anos. A incidência de obesidade entre as mulheres da zona rural aumentou a partir dos 40 anos e diminuiu drasticamente após os 60 anos. As prevalências brutas de obesidade foram de $19,9 \%$ para a zona urbana e de $23,8 \%$ para a rural. As prevalências padronizadas de sobrepeso, para as zonas urbana e rural, foram de $34,7 \%$ e $34,2 \%$, respectivamente.

A obesidade parece ser fundamentalmente um problema de saúde na mulher. Verificando-se as diferenças de prevalência segundo gênero e idade (Tabela 3), para todas as idades a obesidade foi menos freqüente no sexo masculino do que no feminino.

\section{I S C U S S Ã O}

As prevalências de excesso de peso e de obesidade foram relativamente altas entre as mulheres, principalmente na zona rural. A tendência ao excesso de peso naquelas pertencentes aos grupamentos mais pobres de populações urbanas tem sido demonstrada em diversos trabalhos ${ }^{1,2,7,16,17}$. Destaca-se que foi sensivelmente mais alta a prevalênica da obesidade entre as mulheres pertencentes à amostra deste estudo, em comparação com a encontrada na PNSN de $1989^{6}$ e na pesquisa de M onteiro \& Conde (1999) 8 . Provavelmente, a alta prevalência de excesso de peso no grupo populacional feminino, principalmente na zona rural, e as demais doenças crônicas a ele relacionadas são decorrentes do modo de vida da população, caracterizando um perfil epidemiológico de transição.

Tabela 2. Número e porcentagem de mulheres segundo índice de massa corporal, zona de residência e idade. Municípios de Monteiro Lobato e Santo Antônio do Pinhal, 1997.

\begin{tabular}{|c|c|c|c|c|c|c|c|c|c|c|}
\hline \multirow{3}{*}{ Categoria } & \multicolumn{6}{|c|}{ Anos } & \multicolumn{4}{|c|}{ Total } \\
\hline & \multicolumn{2}{|c|}{$20-39$} & \multicolumn{2}{|c|}{$40-59$} & \multicolumn{2}{|c|}{$\geq 60$} & \multicolumn{2}{|r|}{$\mathrm{PB}^{1}$} & \multirow{2}{*}{$\begin{array}{c}\mathrm{PP}^{2} \\
\%\end{array}$} & \multirow{2}{*}{$\frac{\text { IC95 (PP) }}{\%}$} \\
\hline & $n$ & $\%$ & $\mathrm{n}$ & $\%$ & $\mathrm{n}$ & $\%$ & $\mathrm{~N}$ & $\%$ & & \\
\hline \multicolumn{11}{|l|}{ Urbana } \\
\hline Sem excesso IMC $<25$ & 44 & 56,4 & 15 & 33,3 & 13 & 34,2 & 72 & 44,7 & 45,5 & $(38,5-52,5)$ \\
\hline Pré-obesidade $25,0>=\mid M C<30,0$ & 20 & 25,6 & 20 & 44,4 & 17 & 44,7 & 57 & 35,4 & 34,7 & $(28,7-41,7)$ \\
\hline Obesidade $\mid \mathrm{MC}>=30$ & 14 & 17,9 & 10 & 22,2 & 8 & 21,1 & 32 & 19,9 & 19,8 & $(14,2-25,4)$ \\
\hline Subtotal & 78 & 48,4 & 45 & 28,0 & 38 & 23,6 & 161 & & & \\
\hline \multicolumn{11}{|l|}{ Rural } \\
\hline Sem excesso IMC $<25$ & 70 & 49,0 & 23 & 26,7 & 16 & 50,0 & 109 & 41,8 & 42,4 & $(35,9-49,4)$ \\
\hline Pré-obesidade $25,0>=\mid M C<30,0$ & 41 & 28,7 & 39 & 45,3 & 10 & 31,3 & 90 & 34,5 & 34,2 & $(27,2-41,2)$ \\
\hline Obesidade $\mid \mathrm{M} C>=30$ & 32 & 22,4 & 24 & 27,9 & 6 & 18,8 & 62 & 23,8 & 23,4 & $(17,4-30,4)$ \\
\hline Subtotal & 143 & 54,8 & 86 & 32,9 & 32 & 12,3 & 261 & & & \\
\hline
\end{tabular}

Nota: (1) PB = Prevalência Bruta; (2) PP = Prevalência padronizada; (3) IC95 = Intervalo de confiança de $95 \%$.

Tabela 3. Prevalência de obesidade, segundo gênero e idade. Municípios de Monteiro Lobato e Santo Antônio do Pinhal, 1997.

\begin{tabular}{|c|c|c|c|c|c|c|c|c|c|c|c|c|c|c|c|c|c|c|c|}
\hline \multirow{2}{*}{ Gênero } & \multicolumn{3}{|c|}{ 20-29 anos } & \multicolumn{3}{|c|}{ 30-39 anos } & \multicolumn{3}{|c|}{ 40-49 anos } & \multicolumn{3}{|c|}{ 50-59 anos } & \multicolumn{3}{|c|}{$\geq 60$ anos } & \multicolumn{4}{|c|}{ Total } \\
\hline & $\mathrm{n}$ & $\mathrm{C}$ & $\%$ & $\mathrm{n}$ & $c$ & $\%$ & $n$ & $C$ & $\%$ & $\mathrm{n}$ & $\mathrm{C}$ & $\%$ & $n$ & C & $\%$ & $\mathrm{~N}^{1}$ & $C^{2}$ & $\mathrm{~PB}^{3}$ & $\mathrm{PP}^{4}$ \\
\hline Hor & 87 & 2 & 2,3 & 92 & 4 & 4,3 & 65 & 4 & 6,2 & 54 & 6 & 11,1 & 62 & 4 & 6,5 & 360 & 20 & 5,6 & 5,6 \\
\hline Mulheres & 115 & 16 & 13,9 & 106 & 30 & 28,3 & 70 & 19 & 27,1 & 61 & 15 & 24,6 & 70 & 14 & 20,0 & 422 & 94 & 19,9 & 21,6 \\
\hline Total & 202 & 18 & 8,9 & 198 & 34 & 17,2 & 135 & 23 & 17,0 & 115 & 21 & 18,3 & 132 & 18 & 13,6 & 782 & 114 & & 14,6 \\
\hline
\end{tabular}

Nota: (1) $\mathrm{N}=$ indivíduos na faixa etária; (2) $\mathrm{C}=$ número de casos; (3) PB = Prevalência Bruta; (4) PP = Prevalência padronizada. 
Também o período curto entre os partos deve ter contribuído para o excesso de peso observado no sexo feminino, pois a taxa de fecundidade nos municípios considerados no presente trabalho foi superior à encontrada no Estado de São Paulo na década de $80^{18}$, o que deve ter se repetido nos anos posteriores.

Por outro lado, as atividades da mulher da zona rural eram tradicionalmente voltadas à lida com animais em currais e ao cultivo de hortas no entorno da residência. Com as mudanças ocorridas na ocupação do homem, que, por exemplo, de antigo proprietário se transformou em caseiro, este tem se apropriado dessas tarefas. Assim, a vida sedentária imposta à mulher rural deve ser um outro importante fator de risco para a obesidade encontrada nesse grupo ${ }^{18}$.

O trabalho feminino da população pobre na zona urbana é geralmente voltado ao serviço doméstico, predominando as categorias de donas de casa, empregadas domésticas e faxineiras. As residências têm poucos cômodos, não raramente quarto e sala, abrigando marido, esposa e filhos. Nessas condições, a dona de casa tem pouco espaço para a atividade física ${ }^{15}$.

Além disso, outros achados nesta população devem ser apontados. A pesar da alta prevalência de obesidade, mesmo entre as mulheres mais jovens, o padrão alimentar deste grupo sofreu poucas mudanças em relação à dieta do "caipira" tradicional do Estado de São Paulo, baseando-se na ingestão diária de arroz, feijão e verduras, sendo o consumo de carne apenas ocasional. As únicas mudanças observadas foram a substituição da gordura de porco por óleo vegetal e o fato de a dieta não se caracterizar por alta densidade energética ${ }^{19}$. Verificou-se também que neste grupo não ocorreu aumento significativo da estatura dos adultos nas três últimas décadas ${ }^{20}$. Adicionalmente, registrou-se alta prevalência de baixa estatura nos filhos das obesas, reforçando a hipótese de existência de nexo entre baixa estatura e obesidade ${ }^{21}$.
A este respeito, os resultados do estudo de Velásquez-M eléndez (1999) ${ }^{12}$, com população da área metropolitana de São Paulo, demonstraram associação da baixa estatura com obesidade centralizada e dislipidemias em mulheres, indicando a existência de vínculo entre desnutrição pregressa e obesidade.

Depreende-se pelos diferentes fatores envolvidos no crescimento da prevalência do excesso de peso, nas mais diversas populações, que a história natural da obesidade é complexa e pouco conhecida, devendo ser alvo de novos estudos.

\section{A GRA DECIMENTOS}

Pesquisa Financiada pela FA PESP (Fundação de Amparo à Pesquisa do Estado de São Paulo), proc. n.o 96/08081-2.

\section{REFERÊ N C IAS}

1. Haffner SM, Stern MP, Mitchell BD, Hazuda HP. Predictors of obesity in Mexican Adults. Am J Clin Nutr 1991; 53:1571S-6S.

2. Delpeuch F, Maire B. Obesity and developing countries of the South. Med Trop 1997; 57:380-8.

3. Sundsquit J, Malmstrom M, Johanson SE. Cardiovascular risk factors and the neighbourhood environment: a multilevel analysis. Int J Epidemiol 1999; 28:445-9.

4. Sarlio-Lahteenkorva S, Lahelma E. The association of body mass index with social and economic disadvantage in women and men. Int J Epidemiol 1999; 28:445-9.

5. Instituto Brasileiro de Geografia e Estatística. Metodologia do estudo nacional de despesa familiar - Endef. Rio de Janeiro; 1983. (M imeografado).

6. Instituto Nacional de Alimentação e Nutrição. Pesquisa Nacional sobre Saúde e Nutrição (PNSN) - 1989. Arquivo da Pesquisa. Brasília; 1990. (M imeografado). 
7. Martins IS, M eléndez-Velásquez GG, Cervato AM . Estado Nutricional de grupamentos sociais da área metropolitana de São Paulo. Cad Saúde Pública 1999;15:71-8.

8. Monteiro CA, Conde VL. A tendência secular da obesidade segundo estratos sociais: Nordeste e Sudeste do Brasil, 1975-1989-1997. Arch Bras Endocrinol Metab 1999; 43:186-94.

9. Popkin BM , Keyou G, Zhai F, Guo X, M a H, Zoohori $N$. The nutrition transition in China: a cross-sectional analisys. Am J Clin Nutr 1993; 7:333-46.

10. Monteiro CA, M ondini L, Souza ALM, Popkin BM . Da desnutrição para a obesidade: a transição nutricional no Brasil. In: Monteiro CA. Velhos e novos males da saúde no Brasil: a evolução do país e suas doenças. São Paulo: Hucitec; 1995. p.247-55.

11. World Health Organization. Previnting and managing the global epidemic of obesity. Report Geneve; 1995. (Technical Report Series, 854).

12. Velásquez-M eléndez GG, M artins IS, Cervato $A, M$, Coelho LT, Marucci MFN. Central obesity and mass index with stature relationship in São Paulo Metropolitan Area. Int J Obes 1999; 23:639-44.

13. Mondini L. Desnutrição e obesidade no Brasil: relevância epidemiológica e padrões de distribuição intra-familiar em diferentes extratos econômicos e regionais São Paulo; 1996 [Tese]. Faculdade de Saúde Pública, USP.

14. Secretaria de Desenvolvimento e Assistência Social. Zonas de pobreza 2000. (Mimeografado).

15. M artins IS. Hábitos relacionados ao estilo de vida: tabagismo, etilismo e sedentarismo. In: Martins,
IS, Oliveira DC, Fischer FM. Saúde, educação e trabalho nos Municípios de Monteiro Lobato e Santo Antônio do Pinhal. 1999. São Paulo; 1999. Relatório Final FAPESP. Cap. 3.

16. Coitinho DC, Leão $M M$, Recine $E$, Sichieri R. Condições nutricionais da população brasileira: adultos e idosos In: Instituto Nacional de Alimentação e Nutrição. Pesquisa Nacional sobre Saúde e Nutrição Brasília: Ministério da Saúde; 1991.

17. Duncan BB, Schmidt MF, Achutti AC, Polansczyk CA, Benia LR, Maia AA. Socioeconomic distribution of noncommunicable disease: the case of Porto Alegre. Bull Pan Am Health Organ 1993; 27:337-43.

18. Perestrelo JP. Demografia, economia e história: trajetória dos municípios de Santo Antônio do Pinhal e Monteiro Lobato, SP. In: Martins IS, Oliveira DC, Fischer FM. Saúde, educação e trabalho nos Municípios de Monteiro Lobato e Santo Antônio do Pinhal. 1999. São Paulo; 1999. Relatório Final FAPESP. cap. 1. p.1-53.

19. M artins IS, Oliveira, DC, Perestrelo JPP, M arinho SP. Obesity and nutritional transition in impoverished segments of Brazilian Society. Ann Nutr Metab 2001; 45:(Suppl 1):406.

20. Perestrelo JPP, Martins IS, M arinho SP, Oliveira DC. Questioning the secular tendency of growth. Ann Nutr M etab 2001; 45(Suppl 1):412.

21. M arinho SP, M artins IS, Perestrelo JPP, Oliveira DC. Comparing the nutritional status of parents and children. Ann Nutr M etab 2001; 45:(Suppl 1):455.

Recebido para publicação em 18 de junho de 2001 e aceito em 5 de setembro de 2002 . 